\title{
Comparisons between centrifuge and numerical modeling results for slope toppling failure
}

\author{
CHEN ZuYu ${ }^{1,4}$, GONG WenJun ${ }^{1 *}$, MA GuoWei ${ }^{2}$, WANG Jie ${ }^{3}$, HE Lei $^{5}$, XING YiChuan ${ }^{4}$ \\ \& XING JianYing ${ }^{6}$ \\ ${ }^{1}$ School of Transportation Science and Engineering, Beihang University, Beijing 100191, China; \\ ${ }^{2}$ School of Civil and Resource Engineering, University of Western Australia, 35 Stirling Highway, Crawley WA 6009, Australia; \\ ${ }^{3}$ Institute of Mechanics, Chinese Academy of Sciences, Beijing 100190, China; \\ ${ }^{4}$ Department of Geotechnical Engineering, China Institute of Water Resources and Hydropower, Beijing 100048, China; \\ ${ }^{5}$ Department of Civil Engineering, Monash University, Building 60, Clayton VIC 3800, Australia; \\ ${ }^{6}$ Yellow River Engineering Consulting Co., Ltd., Zhengzhou 450003, China
}

Received April 15, 2015; accepted July 1, 2015; published online July 14, 2015

\begin{abstract}
This paper presents series studies on the toppling mechanism by centrifuge tests and numerical simulations. Two different discrete element methods, i.e., the continuum-based discrete element method (CDEM) and the discontinuous deformation analysis (DDA), are adopted. The modeling results show that both the methods can accurately capture the failure modes of the centrifuge tests, including three distinct zones and two failure surfaces. Comparisons are made between the physical test and numerical simulation results. The critical inclination angle of the tilting table where the slope models are fixed on can be moderately predicted by the two methods, with different degrees of precision. The error between the test results and the simulated results is within $1 \%$ for the slope models without rock-bridges by both CDEM and DDA. However, it is amplified for the staggered-joint models that simulate the rock-bridges. With DDA, the average error is about 5\%, and the maximum error is up to $17 \%$. While with CDEM, the errors for the aligned-joint models are ranged from $1 \%$ to $6 \%$, and it is from $10 \%$ to $29 \%$ for the staggered-joint models. The two numerical methods show the capability in simulating toppling failure of blocky rock mass with and without rock-bridges. The model with rock-bridges which provides a certain bending resistance is more stable than the one without any rock-bridge. In addition, the two failure surfaces were observed, which is different from the common understanding that only one failure surface appears.
\end{abstract}

rock slope model, toppling failure, rock-bridge, centrifuge test, continuum based discrete element method, discontinuous deformation analysis

Citation: Chen Z Y, Gong W J, Ma G W, et al. Comparisons between centrifuge and numerical modeling results for slope toppling failure. Sci China Tech Sci, 2015, 58: 1497-1508, doi: 10.1007/s11431-015-5889-x

\section{Introduction}

Toppling is a typical rock slope failure mode commonly encountered in nature and engineering practice. Physical and numerical modeling is an important approach to under-

*Corresponding author (email: gwj6641118@buaa.edu.cn) standing the mechanism and susceptibility of rock slope toppling failure.

In the area of physical modeling, the base friction models and tilt tables were popular in the 1970s and early 1980s [1-4]. However, these models were limited to their smallscale that are unable to reproduce the stress state represented by the field. Centrifuge modeling is useful for studying 
prototype behavior in a model scale where the mechanisms involved are mainly governed by gravity induced body forces. Recent work on centrifuge modeling for rock toppling include Adhikary et al. [5] who investigated flexural toppling in foliated rock slopes and Zhang et al. [6] on the extended Goodman Bray method accommodating nonpersistent basal planes of rock columns.

Discontinuum-based methods such as distinct element code (UDEC) and discontinuous deformation analysis (DDA), by contrast, have achieved great success in accounting for the kinematics of discrete blocks and have been widely used to investigate the toppling failure mechanisms of rock slope [7-14]. However, all the analyzed rock masses are assumed to be divided by fully-persistent joints, fracturing through intact rock-bridge is not taken into consideration. In order to accurately predict rock slope failure, including both fracturing through intact rock mass and kinematic behavior of a failed rock slope, a lot of methods have been developed. Pritchard and Savigny [7] used UDEC to model the failure mechanism of flexural toppling. A linear elastic-plastic constitutive relationship was assigned to blocks, when the strength of the block material was reached, slope fracture failure occurred. However, Alzo'ubi et al. [15] failed to predict the location of the failure surface of centrifuge tests using the same method. They further adopted an improved UDEC with a Voronoi joint generator which made fracturing through intact rock mass directly to model the centrifuge tests, the results showed that the new method could accurately predict both the location of the failure surface and the failure centrifugal acceleration. The discontinuum-based methods with discrete blocks or particles bounded by joints has also been successfully used in other rock engineering region [16-18]. In addition, some continuum-discontinuum methods were developed to address the failure problems recently. Eberhardt et al. [19] adopted a hybrid finite element/discrete element method ELFEN to analyze the failure process of the 1991 Randa rockslide. Zhang et al. [20] successfully predicted the failure state of centrifuge tests by the numerical manifold method (NMM). Feng et al. [21] used the continuum-based discrete element (CDEM) to analyze Jiweishan landslide.

This paper reports a well organized centrifuge testing plan for toppling failure analysis with the following highlights.

(1) The model is placed on a tilting table that rotates at a particular gravitational force until the inclination of the table is big enough to make the slope toppling. Therefore, every test is modeled in a limit equilibrium state. Combinations of various gravitational forces and tilting angles at failure realize the idea of 'modeling of models' [22,23] in centrifuge tests.

(2) The rock slope is modeled by stacked aluminum and plastic cubic blocks that are manufactured in accordance with professional standards, thus allowing uniform geometry and material properties, suitable for validation purposes with numerical approaches.

(3) The aluminum and plastic cubic blocks represent the models with and without bending resistance respectively. The findings have particular significance in understanding the toppling mechanism that should be considered in a numerical method.

In their report on a comprehensive review of the use of centrifuge modeling for rock slopes, Chen at al. [24] briefly introduced the results of this test plan. This paper continues to present the research outcomes that compare the testing results with the continuous-discontinuous method CDEM and DDA method.

\section{Centrifuge tests}

\subsection{Test apparatus}

This series of model tests were conducted on the $450 \mathrm{~g}$-t centrifuge facility at China Institute of Water Resource and Hydropower Research (IWHR). A device with a tilting box mounted inside the bucket was developed as shown in Figure 1. A micro motor automatically lifts the tilting box to a required inclination at a specified centrifugal acceleration. Sensors were mounted inside the tilting box to measure the displacements and inclined angle of the sample.

The main technical parameters of the device with the tilting box are as follows.

Size of the container: $300 \mathrm{~mm} \times 300 \mathrm{~mm} \times 600 \mathrm{~mm}$.

Weight of the container: $80 \mathrm{~kg}$.

Maximum payload: $60 \mathrm{~kg}$.

Maximum lifting force: $160 \mathrm{~kg}$.

Maximum tilting angle: $30^{\circ}$.

Maximum acceleration automatically controlled: $30 g$.

Maximum acceleration manually controlled: $100 \mathrm{~g}$.

\subsection{Set-up of the models}

The model slopes were composed of plastic or aluminum cubic blocks, shown in Figures 2(a) and (b), piled up according to a designed layout. The aluminum blocks were manufactured in a workshop with a certain machining pre-

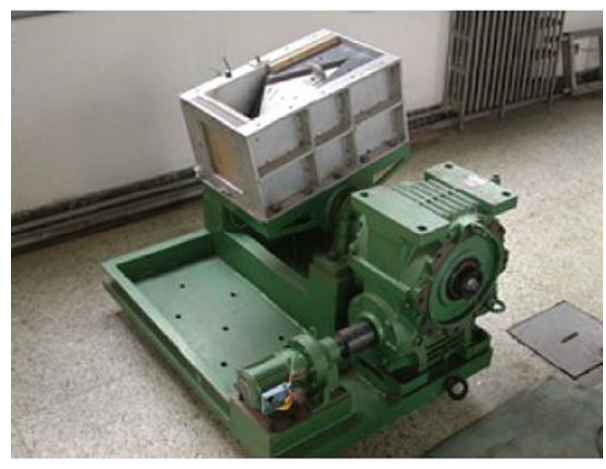

Figure 1 A tilting box in centrifuge [24]. 


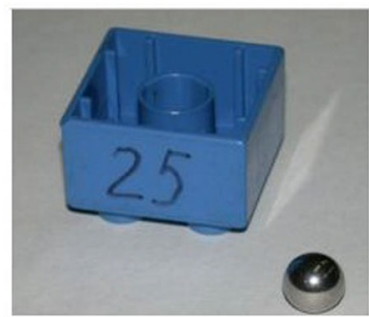

(a)

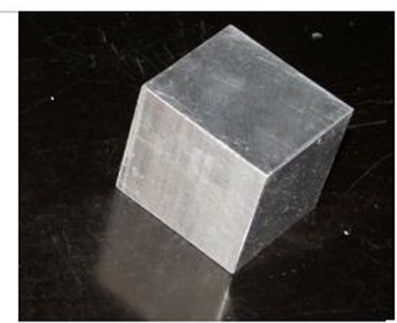

(b)
Figure 2 Cubic blocks. (a) Plastic; (b) aluminum [24].

cision. The plastic blocks were taken from the toys produced by LEGO Corporation.

There is a hole in each plastic block allowing connections with each other, making setting up a model possible. In order to increase the plastic cube's weight, a steel ball was embedded in the cube. The corresponding density is $572 \mathrm{~kg} / \mathrm{m}^{3}$. In the centrifuge tests, the connection between the two adjacent blocks at the hole simulated the tensile resistance produced by the non-persistent joints in the prototype. Two most common rock slope types were considered here, one is coplanar cross-jointed rock slope represented by an aligned-jointed model, the other one is noncoplanar cross-jointed rock slope represented by a stagger-jointed model. Figures 3(a) and (b) show the sketches of the stagger-jointed and aligned-jointed models. In the stagger-jointed model, 10-mm-high plastic cubes were added under every other column. The aluminum model is shown in Figure 3(c). The plastic cube size is $32 \mathrm{~mm} \times 32 \mathrm{~mm} \times 19$ $\mathrm{mm}$. There are two different sizes for the aluminum blocks, i.e., $31 \mathrm{~mm} \times 31 \mathrm{~mm} \times 33 \mathrm{~mm}$ and $29 \mathrm{~mm} \times 29 \mathrm{~mm} \times 29$ $\mathrm{mm}$, with a density of $2.7 \times 10^{3} \mathrm{~kg} / \mathrm{m}^{3}$.

\subsection{Mechanical properties of the testing materials}

Details of the laboratory determination of mechanical properties of model material were reported in Chen et al. [24]. 20 pullout tests were made to measure the possible tensile strength provided by the connection between the plastic cubes and the average pull-out force value is about $19.59 \mathrm{~N}$. Based on the area of the cube, an equivalent tensile strength of $19.1 \mathrm{kPa}$ can be used in the numerical analysis. Five direct shear tests were conducted to determine the shear strength of the aluminum interface. The results show that the cohesion of the interface is small enough to be neglected and the frictional angle is about $13^{\circ}$. Tilting table tests were performed for measuring the sliding property before plastic cubes. The results are that the interface cohesion $c=0.023$ $\mathrm{kPa}$, and the friction angle $\varphi=16.11^{\circ}$.

\subsection{Testing results and discussion}

The models made of plastic and aluminum cubic blocks in the tilting box were tested respectively. The models were put under four different centrifugal accelerations, namely,
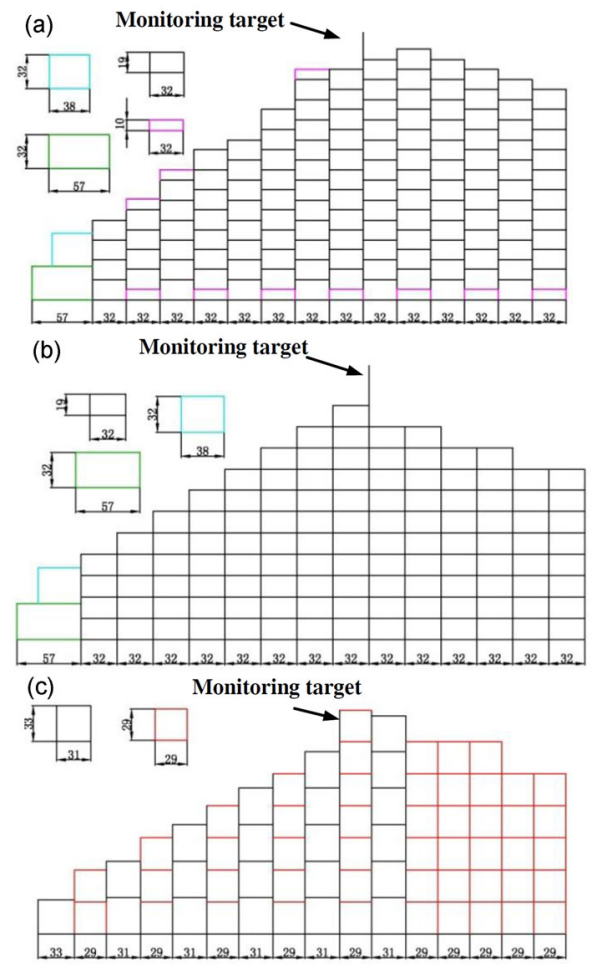

Figure 3 Layout sketches of the models. (a) Stagger-jointed model; (b) aligned-jointed model; (c) aluminum model.

\section{$10,20,30,40 g$ respectively.}

Figure 3 offers detailed sketches of the test failure modes. Figure 4 shows the time versus displacement curve at the monitoring points measured during the tests.

Photos of these failed models and the critically inclined angles of the tilting box under the corresponding centrifugal accelerations are presented in Section 5 in conjunction with those of numerical models for comparison purpose. It can be found that the inclined angles of all the three model slopes decrease with the increase of the centrifugal acceleration. By contrast, under the same centrifugal acceleration, the inclined angle of the plastic-blocky model is about two to three times larger than the inclined angle of the aluminum-blocky model, which means the tensile strength has a certain effect on the stability. However, with the same tensile strength, the inclined angle of the stagger-jointed model is larger than that of the aligned-jointed model. This is largely because that little kinematic release is required for the failure mechanism of the aligned-joined model compared to the stagger-joined model.

It can also be found that a stable zone, a toppling zone and a sliding zone are clearly observed in the aluminum model and the aligned-jointed model, which is in accordance with common sense. However, there is no stable region in the stagger-jointed model. The main reason may be that the critical angle for the stagger-jointed model is large enough that all the block columns topple even for the upper most part of block columns. There is an exception for the stagger-jointed model under $10 g$ centrifugal acceleration. 

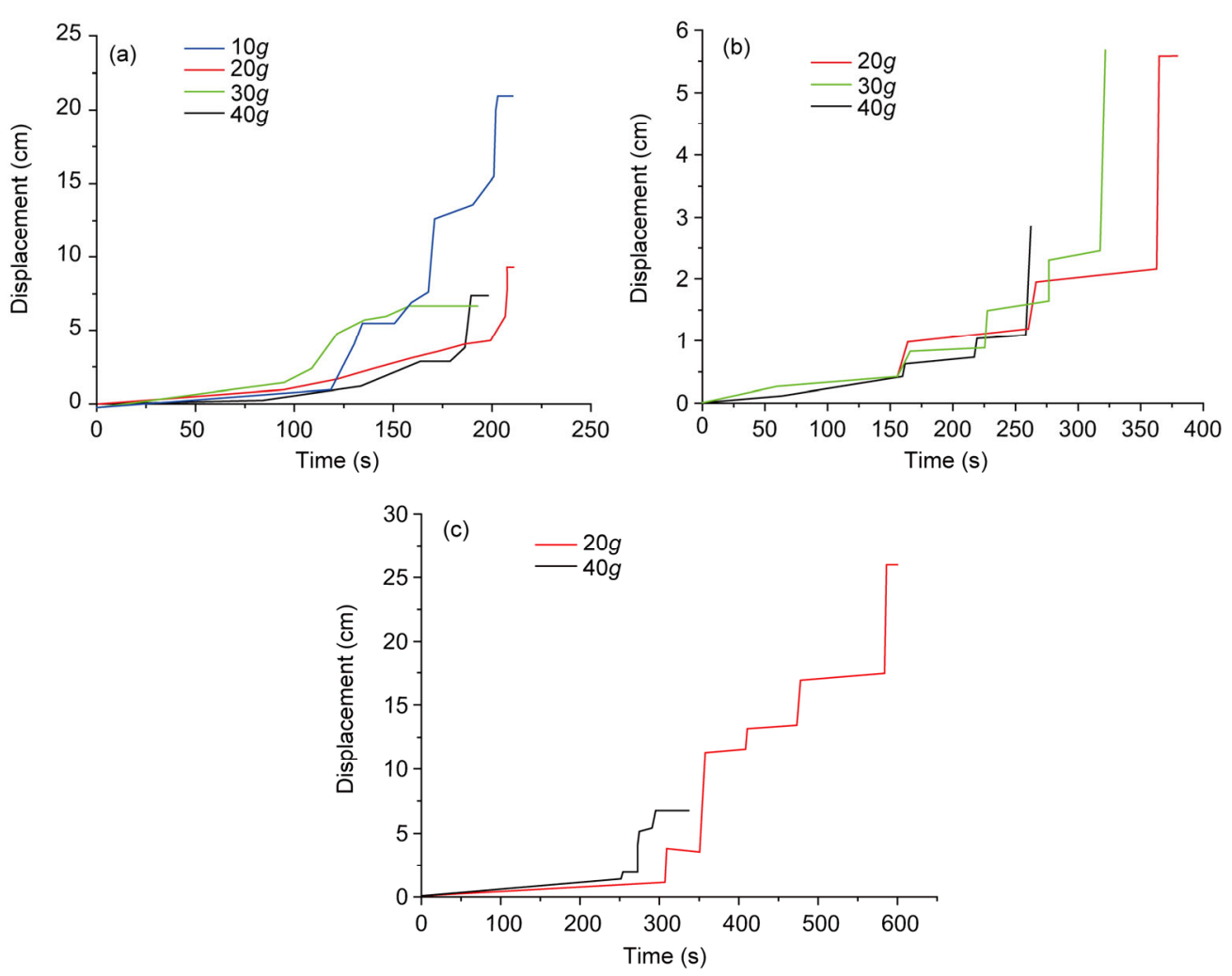

Figure 4 Slope displacement during tests. (a) Aluminum model; (b) aligned-jointed model; (c) stagger-jointed model.

Because the model did not fail even when the inclination of the tilting table approached $37^{\circ} 35^{\prime}$. As the inclined angle exceeded the allowed values range, instead, we gradually increased the rotational speed of the centrifuge till the model failed. At last, the model failed at 17.2g.

In addition, there is also an important finding. Two failure surfaces developed in the blocky columns, the upper one is defined as a 'linear failure surface', above which the blocks tend to slide and fall. The deeper one is defined as a 'deep seated failure surface' which is developed from the toe region and presents a flatter inclination distinguishing the failure region from the whole slope model.

\section{Theoretical background of the numerical methods}

\subsection{The continuum-based element method (CDEM)}

The continuum-based discrete element method (CDEM) developed by the authors $[25-27,21]$ is an explicit approach to simulate the progressive failure of geological mass. This is a combination of finite element method (FEM) and discrete element method (DEM). The forward-difference approximation is adopted to calculate the progressive process through a time marching scheme. As shown in Figure 5, CDEM contains two kinds of elements, blocks and contacts. A discrete block consists of one or more FEM elements, all of which share the same nodes and faces. A contact contains several normal and tangent springs, and each spring owns two nodes which belong to two different blocks. Inside a block, FEM is used, while for contact, DEM is adopted.

The equations of motion are obtained from equilibrium conditions of all forces acting on the nodal masses, resulting in a system of equations of the form

$$
\boldsymbol{M} \ddot{\boldsymbol{u}}+\boldsymbol{C} \dot{\boldsymbol{u}}+\boldsymbol{F}(t)=\boldsymbol{P}(t),
$$

where $\boldsymbol{u}, \dot{\boldsymbol{u}}$ and $\ddot{\boldsymbol{u}}$ denote vectors containing the nodal displacements, velocities and accelerations, respectively; $\boldsymbol{M}$ and $\boldsymbol{C}$ are the mass and damping matrices; the vectors $\boldsymbol{F}(t)$ and $\boldsymbol{P}(t)$ contain the internal and external nodal forces.

In order to avoid the error caused by large rotation, incremental method and strain matrix $\boldsymbol{B}$ are introduced. The complete description of the method can be found in Feng et al. [21].
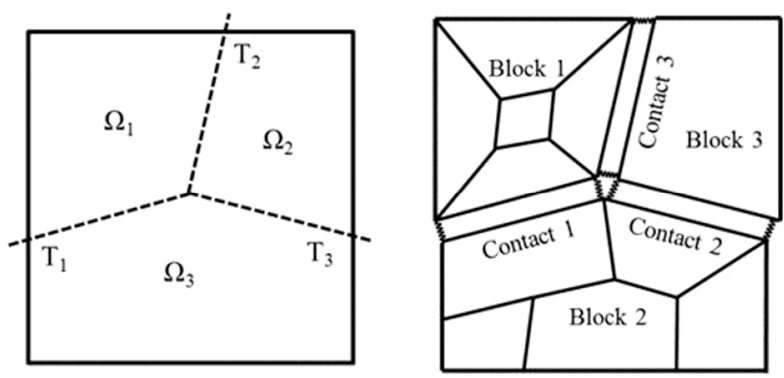

Figure 5 Blocks and contacts in CDEM. 
There are several monitoring points set in the models. If the displacement of the monitoring points increases rapidly during a few minutes, it is believed that the model begins to fail. The CDEM program adopts the following steps to determine the critical angle.

(1) Raising the table to a trial angle $\alpha_{1}$.

(2) Making the model under a certain acceleration which is $n$ times the gravitational acceleration, then calculating the stress and displacement field of the model.

(3) Determining whether the model is failed or not based on the displacement of the monitoring point.

If the model fails under this angel, it indicates that the inclination is greater than the critical one, and the trial angle should be reduced to a new value $\alpha_{2}$. On the other hand, if the model does not fail, the trial angle should be increased to the new value $\alpha_{2}$. The aforementioned steps are repeated with the new value of $\alpha_{2}$. The iteration proceeds until the difference between $\alpha_{1}$ and $\alpha_{2}$ is sufficiently small, say $0.1^{\circ}$.

\subsection{Discontinuous deformation analysis (DDA)}

The DDA method [28] was developed to investigate the kinematics of blocky rock masses. It uses displacements as unknowns and solves the equilibrium equations in the same way as FEM. The formulation is based on dynamic equilibrium that considers the kinematics of individual blocks as well as interactions between blocks. The displacement function for each block is equivalent to the following complete first order approximations of displacements in a twodimensional condition:

$$
\begin{aligned}
& \left(\begin{array}{l}
u \\
v
\end{array}\right)=\left[\begin{array}{cccccc}
1 & 0 & -\left(y-y_{0}\right) & \left(x-x_{0}\right) & 0 & \left(y-y_{0}\right) / 2 \\
0 & 1 & \left(x-x_{0}\right) & 0 & \left(y-y_{0}\right) & \left(x-x_{0}\right) / 2
\end{array}\right] \\
& \left\{\begin{array}{c}
u_{0} \\
v_{0} \\
r_{0} \\
\varepsilon_{x} \\
\varepsilon_{y} \\
r_{x y}
\end{array}\right\} \text {, }
\end{aligned}
$$

where $(x, y)$ are the coordinates of any point within a block, $(u, v)$ are the displacements of a point $(x, y),\left(x_{0}, y_{0}\right)$ are the coordinates of a point within the block (normally the centroid of the block is selected). $u_{0}, v_{0}$ are the rigid body translations at the point $\left(x_{0}, y_{0}\right)$ along $x$ and $y$ directions respectively, $r_{0}$ is the rotation angle in radian around the point $\left(x_{0}, y_{0}\right), \varepsilon_{x}, \varepsilon_{y}$ and $r_{x y}$ are the normal and shear strains of the block.

DDA has the advantage that only 6 degrees of freedom in a $2 \mathrm{D}$ setting for a block are required regardless of the block size and geometry. Benefiting from the simplex integration technique [28,29], DDA derives analytical solutions for the stiffness matrices, the inertia matrices and the force vectors based on a variational potential energy method. The accuracy and efficiency of the DDA method are improved by using the analytical solutions for these matrices [30,31].

A contact model is applied to join and separate the neighboring blocks and a "no penetration, no tension" criterion is obeyed at each contact. The contact effect is represented by applying two stiff contact springs in the normal and shear directions or frictional forces along the sliding edge. The normal and shear contact springs are added if the blocks are in contact and not sliding relative to each other, and deleted if the blocks separate or the normal contact force is tensile. The multi-body contact problem requires a trial-and-error iteration procedure, which was named the open-close iteration by Shi [28].

It is worth highlighting that an implicit time integration algorithm, i.e., the Newmark- $\beta$ method with two parameters $\beta=0.5$ and $r=1.0$ is used in the traditional DDA [32] The numerical damping is essential for the DDA analysis, as it allows the oscillations caused by contact forces to dissipate rapidly, resulting in a stable state, which ultimately allows the open-close iteration to converge rapidly. The amount of numerical damping is also proportional to the time step size.

In this study, the traditional DDA is modified in order to simulate the centrifuge tests of the models. Firstly, a rotating model box with a certain rotating acceleration is added to the discrete block system which is used to simulate the physical centrifuge tests. Secondly the model box can be lifted up at one side gradually so that an inclined slope model under a centrifuge test is simulated. Each increment of the model box inclination angle is small enough so that the inertia force is not introduced into the slope models. The simulated slope model in the model box reaches a stable state at each increment of the inclination angle. The critical angle is obtained when the inclined slope model starts to fall down and then collapse. A tensile failure model is then used for the staggered-joint plastic model. Two different friction angles, i.e., $32.1^{\circ}$ and $16.11^{\circ}$, are adopted for the top/bottom faces and the side faces of cubes of the staggered-joint plastic model, respectively. Other physical parameters are the same as those used in the CDEM simulations.

\section{Representation of typical failure cases}

\subsection{Comparisons: The aluminum models}

\subsubsection{Parameters}

As a typical representation, the CDEM and DDA computation results for aluminum models at $20 \mathrm{~g}$ are described in this section.

The physical properties for aluminum model are: density $\rho=2700 \mathrm{~kg} / \mathrm{m}^{3}$, Poisson's ratio $v=0.34$, Young's modulus $E=68 \mathrm{GPa}$, tensile strength $\sigma_{t}=0.0 \mathrm{kPa}$, friction angle $\varphi=$ $13.1^{\circ}$. They are based on the laboratory test results presented in details in Chen et al. [24]. 
The numerical controlling parameters for CDEM are: time step $\Delta t=1 \times 10^{-6} \mathrm{~s}$, local damping $=0.01$. While those for DDA are: time step size $\Delta t=0.0001 \mathrm{~s}$, maximum displacement ratio $g_{2}=0.0012$, penalty number $k=1020 \mathrm{GPa} / \mathrm{m}$.

\subsubsection{Comparisons between CDEM and physical model}

CDEM starts with a trial angle of $\alpha_{1}=12.2^{\circ}$. Associated with this case, the time versus displacement curve at the monitoring point is shown in Figure 6 that depicts a smooth and virtually horizontal shape, indicating that the model is stable at this trial angle. Similar situation held when $\alpha_{2}$ $=12.3^{\circ}$. However, the displacement of the monitoring point increased rapidly when $\alpha_{3}=12.4^{\circ}$ at $0.25 \mathrm{~s}$ as shown in Figure 6 , thus the critical angle determined by CDEM is $12.4^{\circ}$. Compared to $13.4^{\circ}$ produced by the centrifuge test, the error is $7.4 \%$ for this case. Figure 7 presents the failure process of CDEM at $0.02,0.34,0.38$ and 0.40 s respectively.

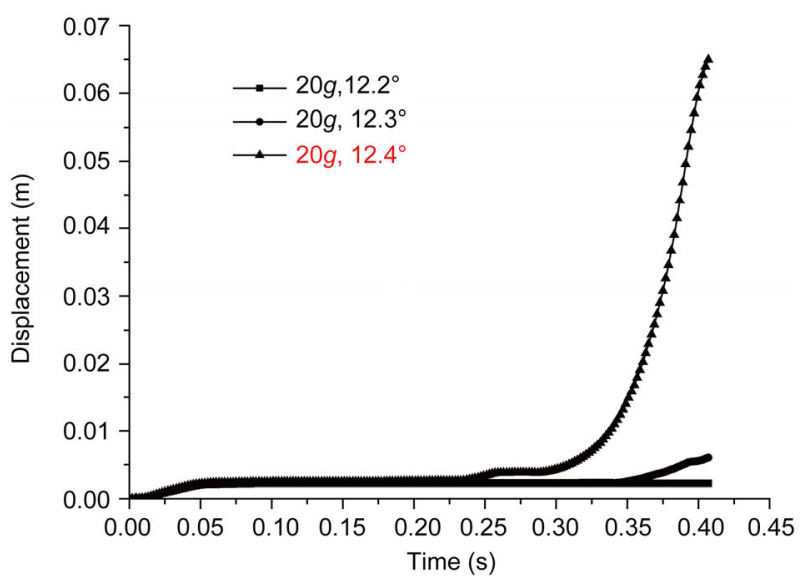

Figure 6 Displacement of monitoring points in aluminum model under $20 \mathrm{~g}$ centrifugal acceleration.
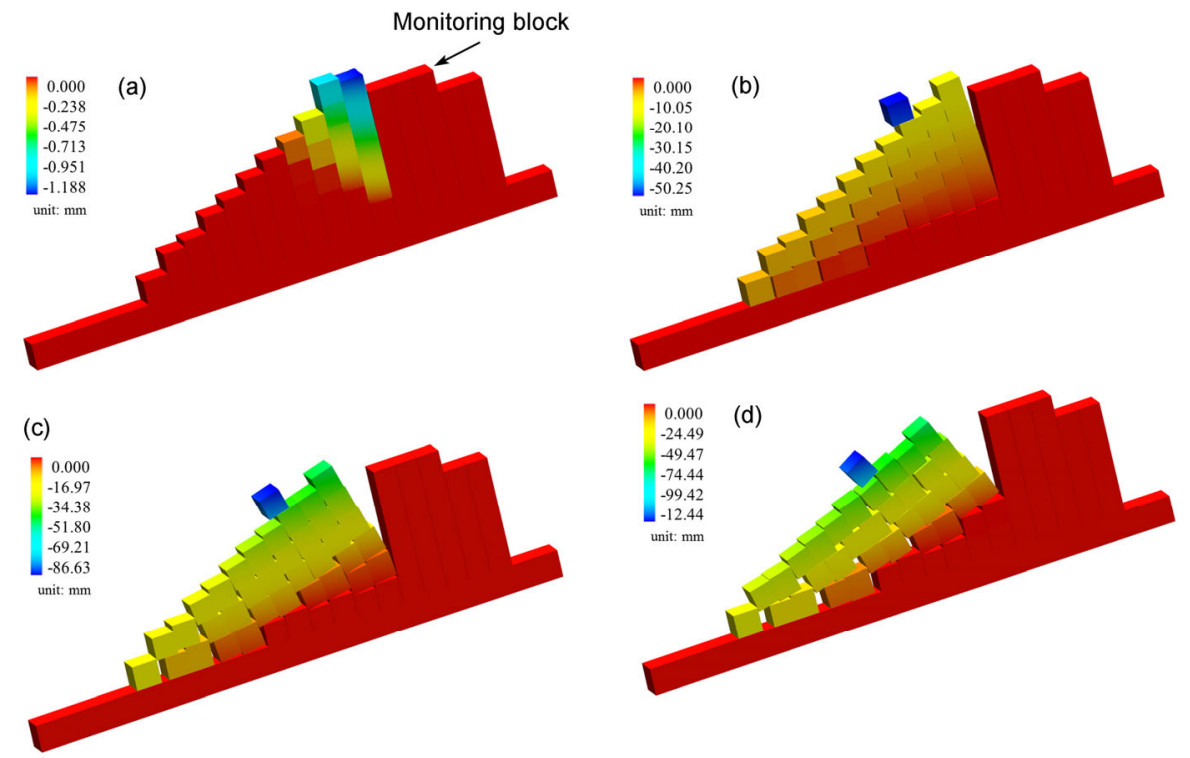

\subsubsection{Comparisons between DDA and physical model}

The DDA simulation is conducted by a two-phase movement of the slope model. An acceleration of $20 \mathrm{~g}$ is first applied to the slope model same as the centrifuge test condition. The slope model is getting stable before the right- hand side is lifted up gradually (Figure 8(a)) with an increment angle of 1/1500 degree per step. Figure 8(b) shows that the slope is still stable when the inclination angle is increased to $10^{\circ}$. The slope starts to slide and topple when the inclination angle of the slope is lifted up to $13.24^{\circ}$ (Figure $8(\mathrm{c})$ ), which gives a critical inclination angle of $13.24^{\circ}$. The final failure pattern is shown in Figure 8(d).

Comparing to the critical angle obtained from the centrifuge experiments, which is $13.4^{\circ}$, DDA simulation gives very good agreement in this aluminum slope model case with an error of only $0.2 \%$. Both sliding failure and toppling failure are observed which also matches well the experimental results.

The final failure modes of the numerical and physical models are compared in Figure 9.

As can be seen from Figure 9, the sizes of the stable zone and failure zone in the physical modes are almost the same with the two numerical results. The gap between the stable zone and the unstable zone is also simulated by the two numerical methods with high precision. In addition, the sliding part on the upper linear failure surface can also be moderately represented by the both methods.

\subsection{Comparisons: the aligned-jointed plastic models}

\subsubsection{Parameters}

Representations of the CDEM and DDA computation for the aligned-jointed plastic models at $30 g$ are given in this

Figure 7 Failure process of aluminum model simulated by CDEM under $20 g$ condition. (a) $0.02 \mathrm{~s}$; (b) $0.34 \mathrm{~s}$; (c) $0.38 \mathrm{~s}$; (d) $0.40 \mathrm{~s}$. 

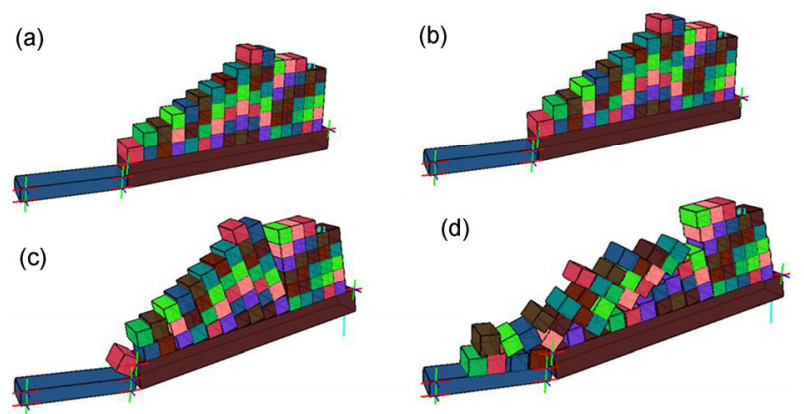

Figure 8 Failure process of aluminum model simulated by DDA under $20 \mathrm{~g}$ condition. (a) $\alpha=0^{\circ}$ at step 6000; (b) $\alpha=10^{\circ}$ at step 21000; (c) $\alpha=$ $13.24^{\circ}$ at step 40000 ; (d) $\alpha=13.24^{\circ}$ at step 60000 .

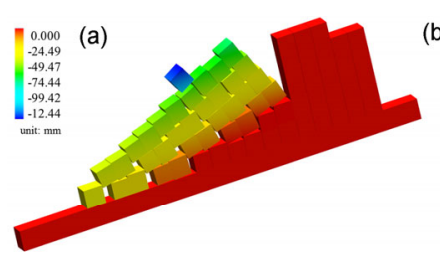

(c)
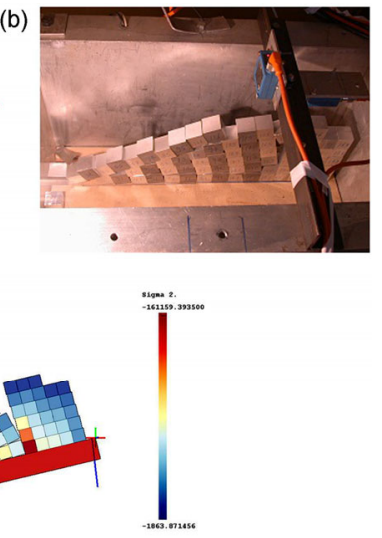

Figure 9 Aluminum model failed under $20 g$ acceleration. (a) CDEM result; (b) physical result; (c) DDA result.

section.

The physical properties for plastic model based on laboratory test results are [24]: density $\rho=572 \mathrm{~kg} / \mathrm{m}^{3}$, Poisson's ratio $v=0.4$, Young's modulus $E=1.2 \mathrm{GPa}$, tensile strength $\sigma_{t}=19.1 \mathrm{kPa}$, friction angle $\varphi=16.1^{\circ}$.

The numerical controlling parameters for CDEM are: time step $\Delta t=1 \times 10^{-6} \mathrm{~s}$, local damping $=0.01$. While those for DDA are: time step size $\Delta t=0.0001 \mathrm{~s}$, maximum displacement ratio $g_{2}=0.0003$, penalty number $k=14.4$ $\mathrm{GPa} / \mathrm{m}$.

\subsubsection{Comparisons between CDEM and physical model}

In order to accurately duplicate the failure process of plastic models, there is an additional parameter named critical tensile displacement $u_{m}$ required for the CDEM method because of the special connection between plastic cubes. If the displacement between the connected cubes is less than the critical displacement, a larger cohesion will be added to the interface between the connected cubes to ensure that sliding will not occur. However, if the displacement is beyond the critical value, the large cohesion will be removed. If the tangential force is large enough, sliding will occur. Here $u_{m}$ $=0.4 \mathrm{~mm}$.

The time versus displacement curves of the four monitoring points are shown in Figure 10. When the trial angle is $25^{\circ}$, the displacement is very small and almost invisible, indicating a stable state. However, when the angle is increased to $25.1^{\circ}$, the displacements of the two monitoring points increase rapidly and is large enough to be visible, which means failure occurs. Thus, the critical angle determined by CDEM is $25.1^{\circ}$, the error is about $16.2 \%$. Figure 11 exhibits the failure processes of the CDEM model.

\subsubsection{Comparisons between DDA and physical model}

For the DDA simulation, an acceleration of $30 \mathrm{~g}$ is first applied to the slope model. Again the slope model is lifted at one side gradually same as the procedure of the centrifuge test. As shown in Figure 12(a), the slope model is stable when the inclination angle is $10^{\circ}$. A minor relative dislocation is formed between the blocky columns at step 35000, when the inclination angel reaches $23.32^{\circ}$ (Figure 12(b)). The dislocation is constantly enlarged with computing progress as shown in Figure 12(c). The final failure pattern is shown in Figure 12(d).

The critical angle of $23.32^{\circ}$ for this case is deviated from the physical result $21.37^{\circ}$ with an error of about $9 \%$.

As noted from Figure 13, the failure modes determined by the two methods match well with the physical failure
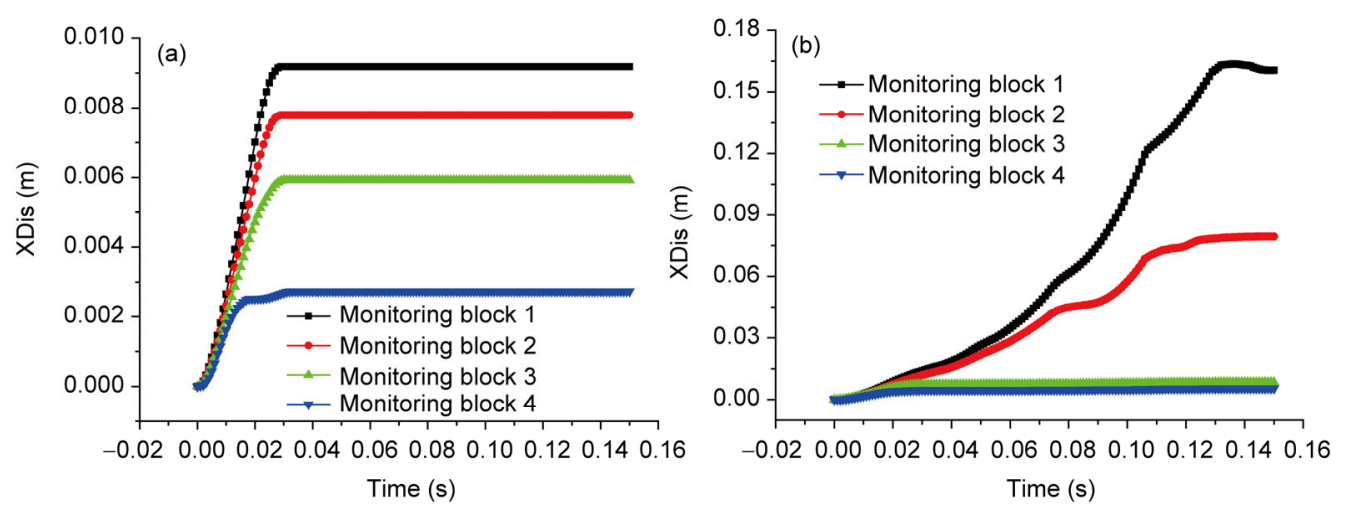

Figure 10 Displacement of monitoring points in aligned-jointed model under $20 \mathrm{~g}$ centrifugal acceleration. (a) $25^{\circ}$; (b) $25.1^{\circ}$. 

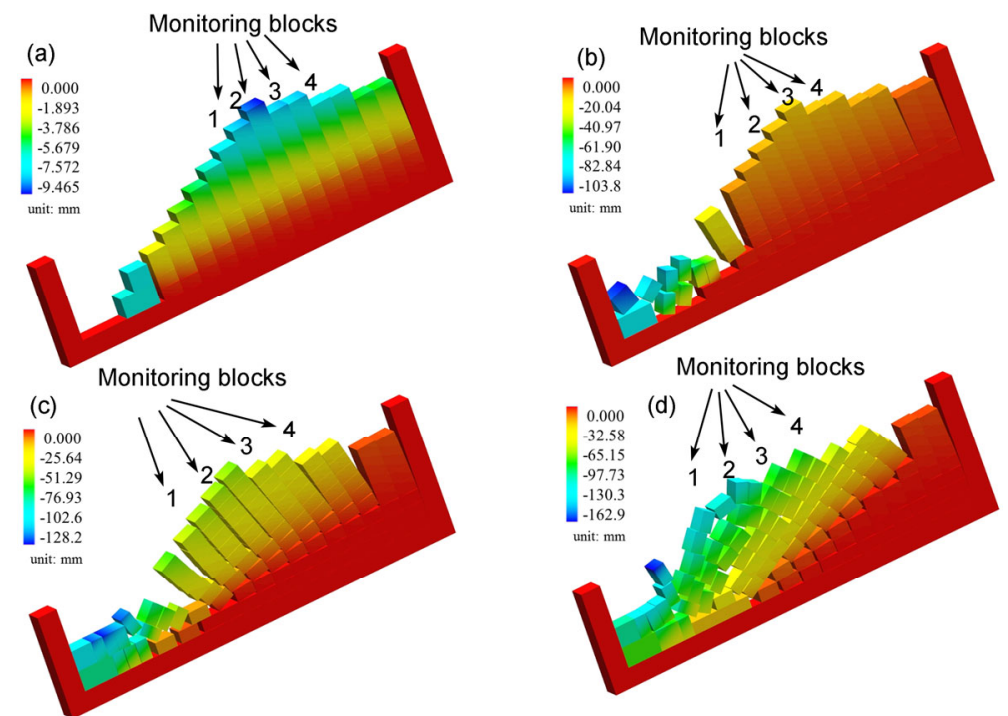

Figure 11 Failure process of aligned-jointed model simulated by CDEM under $30 g$ condition. (a) $0.02 \mathrm{~s}$; (b) $0.08 \mathrm{~s}$; (c) $0.12 \mathrm{~s}$; (d) $0.15 \mathrm{~s}$.

(a)

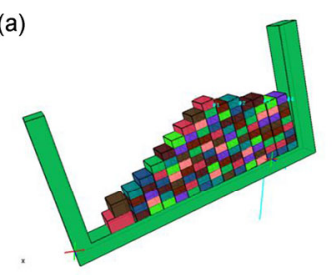

(c)

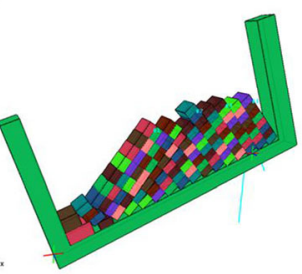

(b)

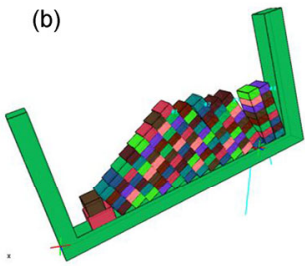

(d)

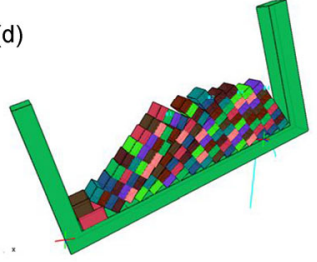

Figure 12 The failure process of aligned-jointed model simulated by DDA under $30 \mathrm{~g}$ condition. (a) $\alpha=10^{\circ}$ at step 2100; (b) $\alpha=23.32^{\circ}$ at step 35000; (c) $\alpha=23.32^{\circ}$ at step 42000 ; (d) $\alpha=23.32^{\circ}$ at step 65000 .

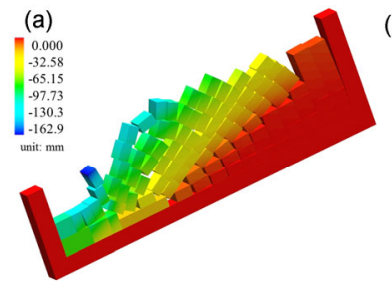

(c)

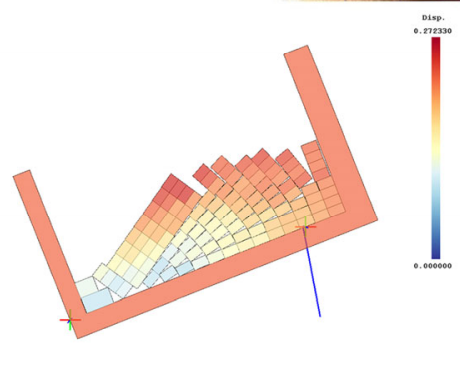

Figure 13 Aligned-jointed model failed under $30 \mathrm{~g}$ acceleration. (a) CDEM result; (b) physical result; (c) DDA result. mode, except for the size of the toppling region determined by DDA, which is slightly larger than the test result.

\subsection{Comparisons: the stagger-jointed plastic models}

\subsubsection{Parameters}

The physical properties and numerical controlling parameters adopted for the stagger-jointed model are the same with the aligned-jointed model.

\subsubsection{Comparisons between CDEM and physical model}

As noted from the time versus displacement curve in Figure 14(a), when the inclination of the tilting table is $31.7^{\circ}$, the movement of the model is small and almost invisible. However, when the angle is $31.8^{\circ}$, large scale movement occurs, as shown in Figure 14(b), indicting toppling failure occurs. Thus, the critical angle determined by CDEM is $31.8^{\circ}$. The relative error for the case is about $14.2 \%$. Figure 15 presents the failure process of the CDEM model.

\subsubsection{Comparisons between DDA and physical model}

Firstly, $20 \mathrm{~g}$ acceleration is set to the slope model same as the centrifugal acceleration. Figure 16(a) shows that the model starts to slide when the inclination angle of the slope reaches $39.38^{\circ}$. Figures 16 (b) and (c) exhibit the two failure processes of the computing model, and Figure 16(d) is the final failure model.

The critical angle determined for the $20 \mathrm{~g}$ case is $39.38^{\circ}$, and the relative error is about $6.3 \%$ comparing to the centrifuge test result $37.05^{\circ}$.

Figure 17 presents the failure mode of the two numerical methods and the physical results. Although the staggered cubes make the failure mechanism more complicated the failure mode of the stagger-jointed model can also be accurately simulated by these two methods. 

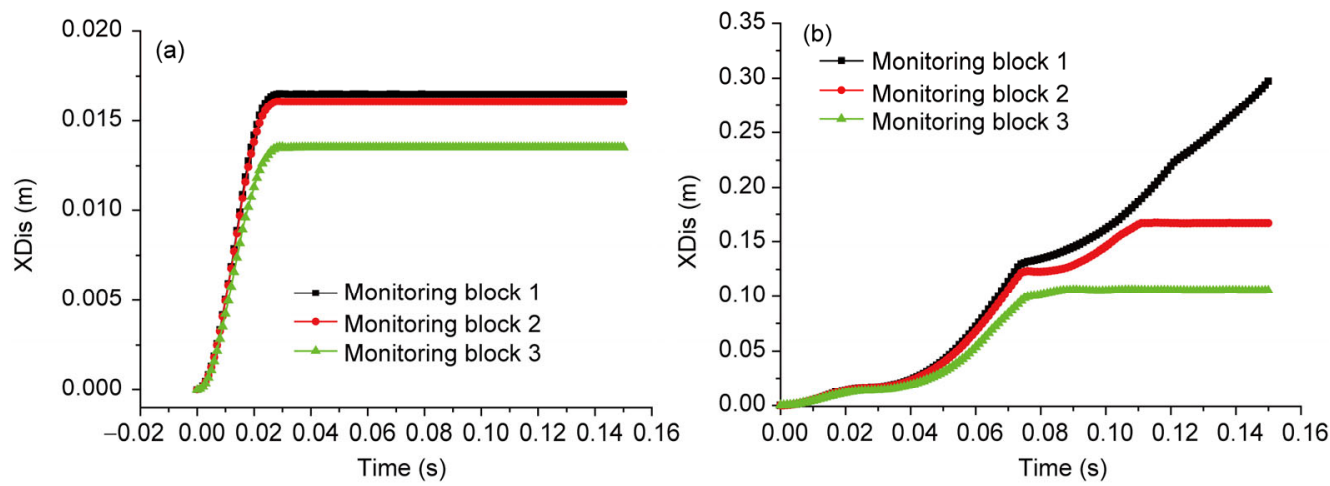

Figure 14 Displacement of monitoring points in stagger-jointed model under $20 \mathrm{~g}$ centrifugal acceleration. (a) $31.7^{\circ}$; (b) $31.8^{\circ}$.
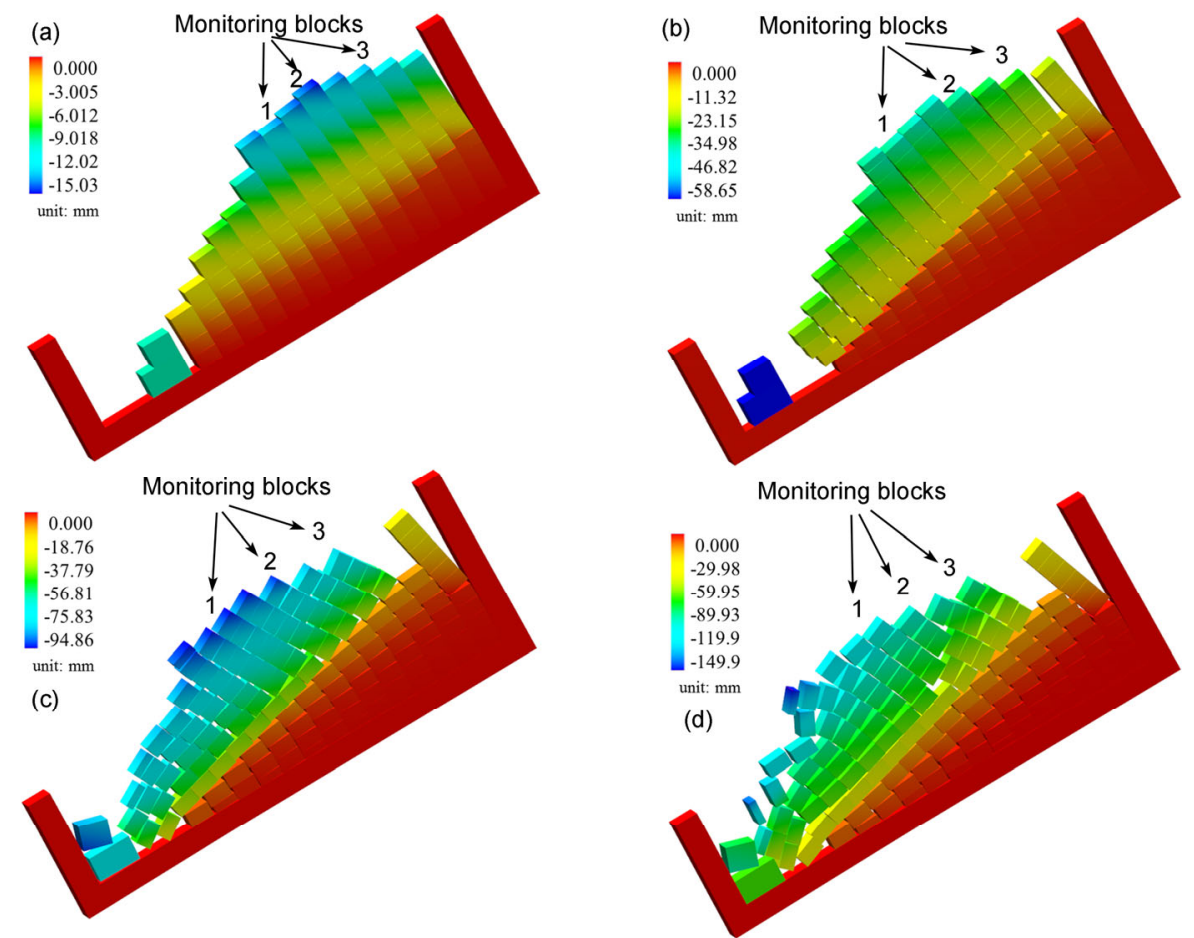

Figure 15 Failure process of stagger-jointed model simulated by CDEM under $20 \mathrm{~g}$ condition. (a) $0.02 \mathrm{~s}$; (b) $0.05 \mathrm{~s}$; (c) $0.07 \mathrm{~s}$; (d) $0.08 \mathrm{~s}$.

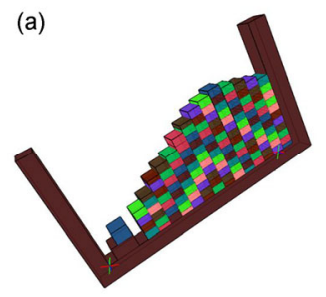

(c)

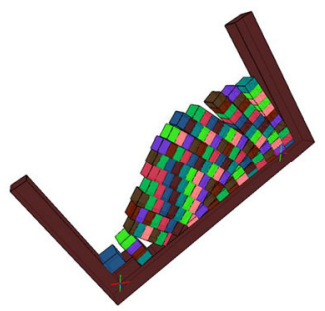

(d)

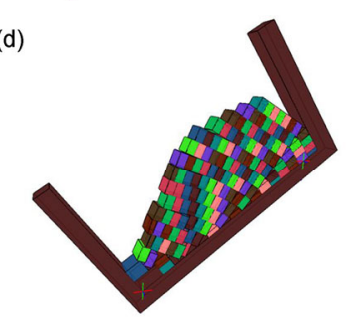

Figure 16 Failure process of stagger-jointed model simulated by DDA under $20 \mathrm{~g}$. (a) $\alpha=39.38^{\circ}$ at step 46000; (b) $\alpha=39.38^{\circ}$ at step 50000; (c) $\alpha=39.38^{\circ}$ at step 55000; (d) $\alpha=39.38^{\circ}$ at step 65000 .
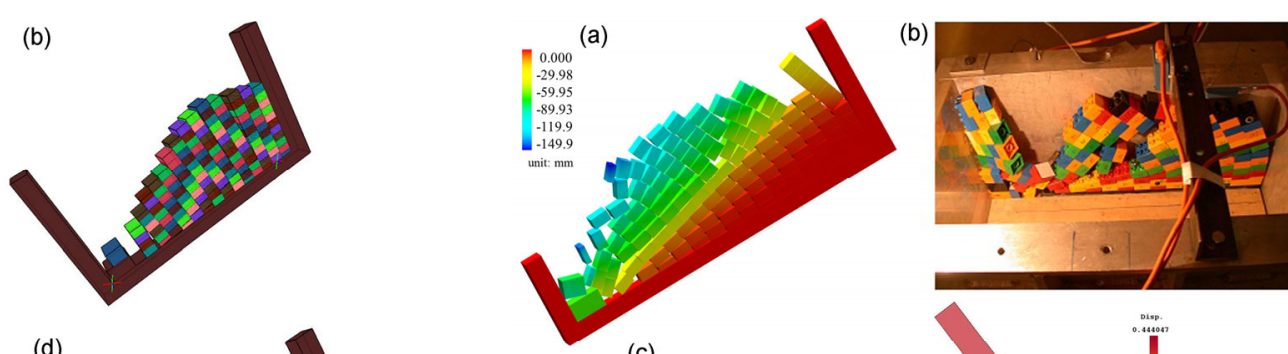

(c)

Figure 17 Stagger-jointed model failed under $20 \mathrm{~g}$ acceleration: (a) CDEM result; (b) physical result; (c) DDA result. 
Table 1 Comparisons of the critical angle between the physical tests and the numerical results

\begin{tabular}{|c|c|c|c|c|c|c|c|c|c|}
\hline \multirow{2}{*}{$\begin{array}{c}\text { Centrifugal } \\
\text { acceleration }(g)\end{array}$} & \multicolumn{3}{|c|}{ Aluminum model } & \multicolumn{3}{|c|}{$\begin{array}{c}\text { Aligned-jointed } \\
\text { model }\end{array}$} & \multicolumn{3}{|c|}{ Stagger-jointed model } \\
\hline & Test & DDA & CDEM & Test & DDA & CDEM & Test & DDA & CDEM \\
\hline 10 & $13^{\circ}$ & $13.23^{\circ}$ & $12.9^{\circ}$ & $31^{\circ} 57^{\prime}$ & $33.28^{\circ}$ & $35.1^{\circ}$ & $37^{\circ} 35^{\prime}$ & - & $37.9^{\circ}$ \\
\hline 20 & $13^{\circ} 38^{\prime}$ & $13.40^{\circ}$ & $12.4^{\circ}$ & $24^{\circ} 12^{\prime}$ & $25.28^{\circ}$ & $25.1^{\circ}$ & $37^{\circ} 05^{\prime}$ & $39.38^{\circ}$ & $31.8^{\circ}$ \\
\hline 30 & $12^{\circ} 35^{\prime}$ & $12.42^{\circ}$ & $12.5^{\circ}$ & $21^{\circ} 37^{\prime}$ & $23.32^{\circ}$ & $22.7^{\circ}$ & $29^{\circ} 50^{\prime}$ & $34.25^{\circ}$ & $26.5^{\circ}$ \\
\hline 40 & $12^{\circ} 50^{\prime}$ & $12.88^{\circ}$ & $12.4^{\circ}$ & $21^{\circ}$ & $23.22^{\circ}$ & $21.3^{\circ}$ & $20^{\circ}$ & $23.99^{\circ}$ & $25.8^{\circ}$ \\
\hline
\end{tabular}

\section{Summary of the 12 cases}

Due to the limited space for presentation, only some representative models were described in Section 4. However the critical angles of the whole set of experiments obtained by centrifuge and the two numerical methods are summarized and compared in Table 1. In addition, photos of all failed models have been given from Figures 18 to 26 .

The error between the test results and the simulated results is within $1 \%$ for the slope models without rock-bridges by both CDEM and DDA. However, it is amplified for the staggered-joint models that simulate the rock-bridges. With DDA, the average error is about $5 \%$, and the maximum error is up to $17 \%$. While with CDEM, the errors for the aligned-joint models are ranged from $1 \%$ to $6 \%$, and it is from $10 \%$ to $29 \%$ for the staggered-joint models. The two numerical methods show the capability in simulating toppling failure of blocky rock mass with and without rockbridges. The model with rock-bridges which provides a certain bending resistance is more stable than the one without any rock-bridge. It is also found that two failure surfaces are developed in the blocky columns which is different from the common understanding that only one failure surface appears.

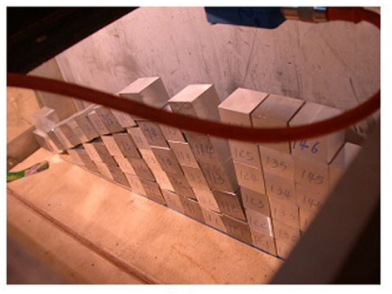

(a)

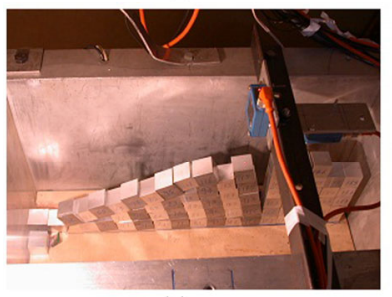

(c)

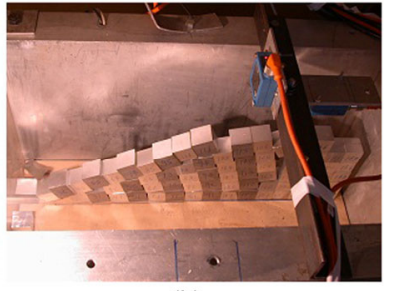

(b)

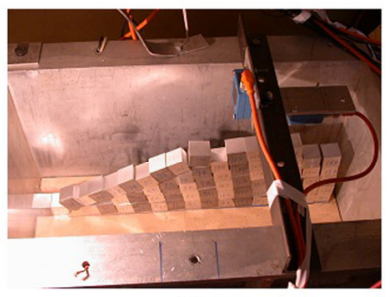

(d)
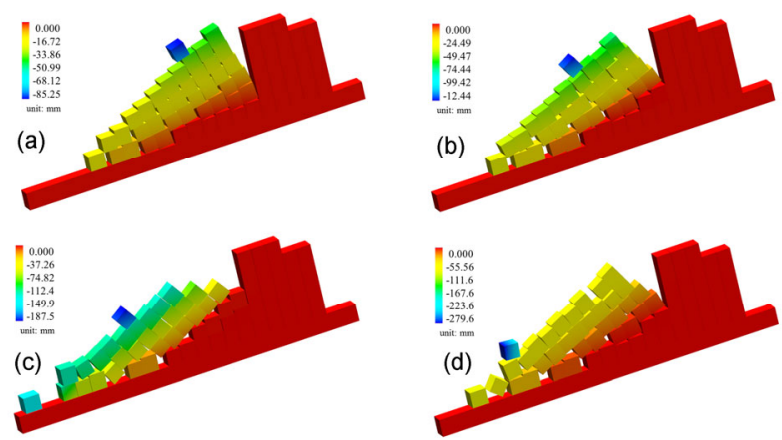

Figure 19 Aluminum model failed simulated by CDEM. (a) $10 g$; (b) $20 g$; (c) $30 g$; (d) $40 g$. (a)

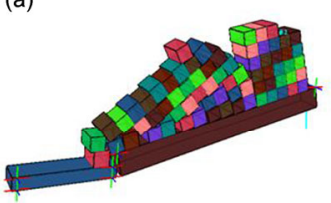

(c)

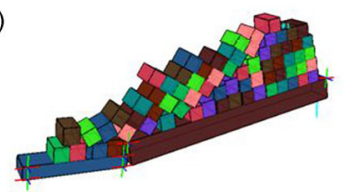

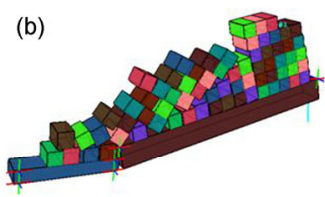

(d)

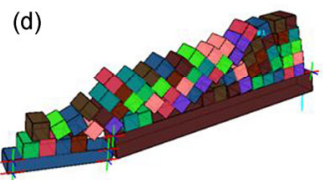

Figure 20 Aluminum model failed simulated by DDA. (a) $10 g$; (b) $20 g$; (c) $30 g$; (d) $40 g$.

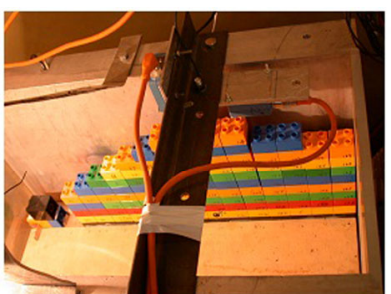

(a)

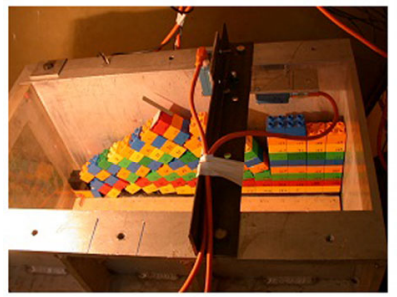

(c)

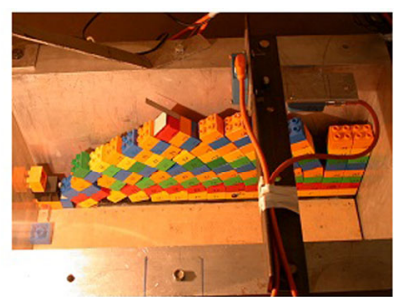

(b)

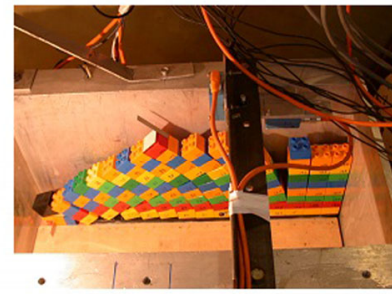

(d)
Figure 18 Pictures of aluminum cubes at failure accelerations. (a) $10 g$; (b) 20g; (c) 30g; (d) 40g [24].
Figure 21 Pictures of aligned-joined model at the failed acceleration. (a) $10 g$; (b) $20 g$; (c) $30 g$; (d) $40 g$ [24]. 

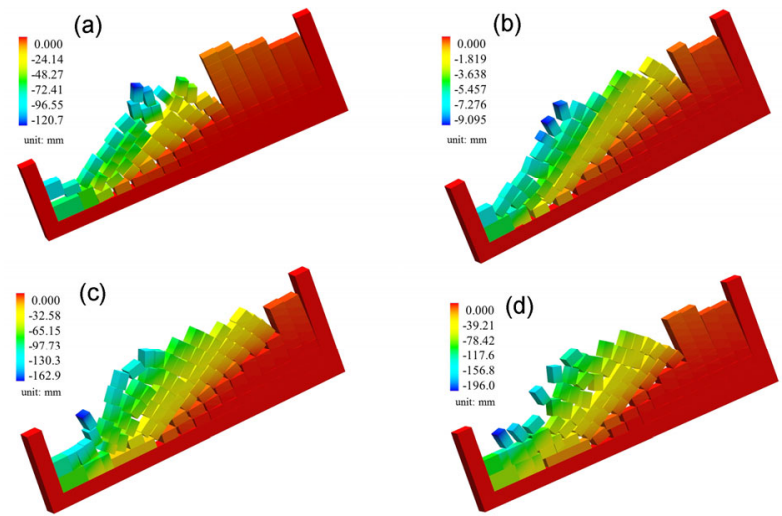

Figure 22 Aligned-jointed model failed simulated by CDEM at the failed acceleration. (a) $10 g$; (b) $20 g$; (c) $30 g$; (d) $40 g$. (a)

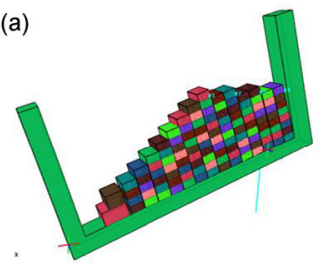

(c)

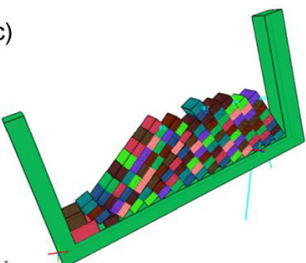

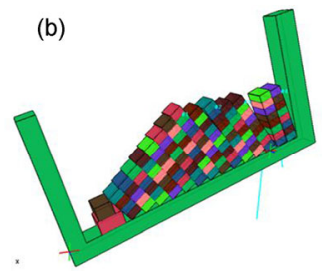

(d)

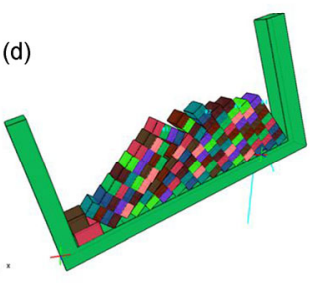

Figure 23 Aligned-jointed model failed simulated by DDA at the failed acceleration. (a) $10 \mathrm{~g}$; (b) $20 \mathrm{~g}$; (c) $30 \mathrm{~g}$; (d) $40 \mathrm{~g}$.

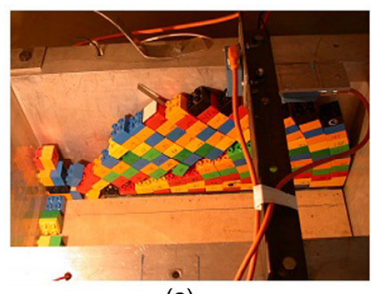

(a)

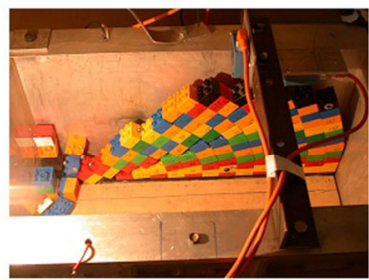

(c)

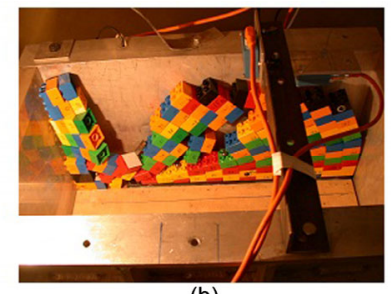

(b)

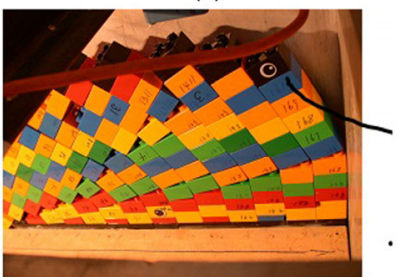

(d)
Figure 24 Pictures of Stagger-jointed model at the failed acceleration. (a) $17.2 \mathrm{~g}$; (b) $20 \mathrm{~g}$; (c) $30 \mathrm{~g}$; (d) $40 \mathrm{~g}$ [24].

\section{Conclusion}

Toppling failures of rock slopes involve both shear and ten-
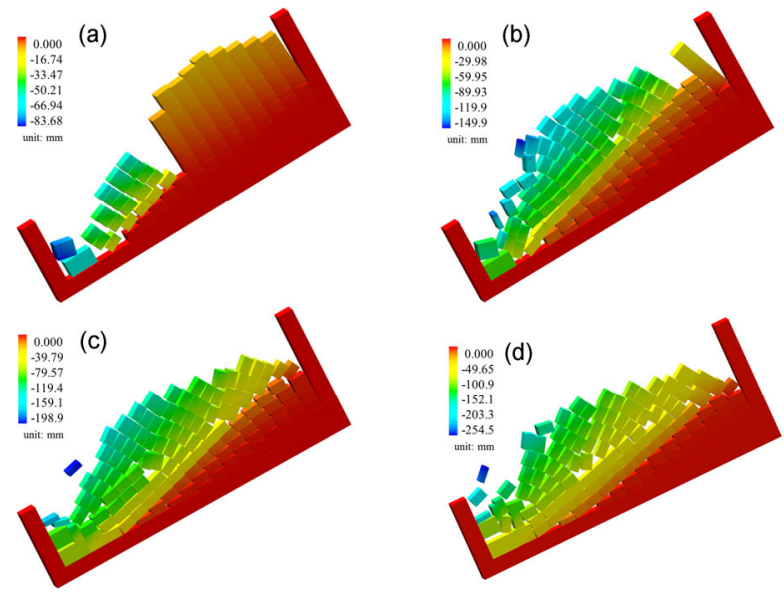

Figure 25 Stagger-jointed model simulated by CDEM at the failed acceleration. (a) $17.2 \mathrm{~g}$; (b) $20 \mathrm{~g}$; (c) $30 \mathrm{~g}$; (d) $40 \mathrm{~g}$.
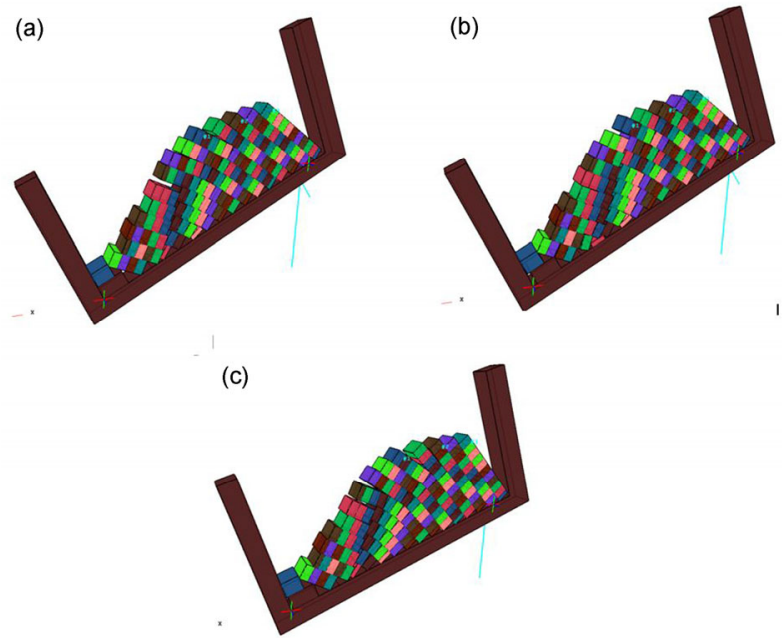

Figure 26 Stagger-jointed model simulated by DDA at the failed acceleration. (a) $20 g$; (b) $30 g$; (c) $40 g$.

sile failures. In order to study the mechanism of toppling, the results of three group model tests in centrifuge are introduced. One is composed of aluminum blocks that is used to study rock slope without bending mechanism. The other two are all composed of plastic blocks but with different arrangement types. The results show that stable zone, toppling zone and sliding zone are clearly observed in the test models. However, different from the common sense that only one failure surface occurs in toppling slope, two failure surfaces appear in all the test models. The upper one was defined as a 'linear failure surface', and the deeper one defined as a 'basal plane'. In addition, the results show that the model with a certain bending resistance is more stable than the model without it.

The CDEM and the DDA numerical methods are adopted to investigate the failure mechanism of the centrifuge tests. The results show that the two numerical methods can accurately determine the critical angle of the centrifuge test, ex- 
cept for the stagger-jointed models which are slightly deviated from the centrifuge tests. In addition, the two methods also moderately capture the failure modes of the centrifuge test, including the three distinct regions and the size of these regions. The striking features that two failure surfaces coexist in one failed model also can be reflected by these two methods.

Thus, the results confirm that both CDEM and DDA are valid and effective in dealing with toppling problems for rock mass with or without rock-bridge.

This work was supported by the National Basic Research Program of China ("973” Project) (Grant No. 2014CB047006).

1 Whyter R J. A study of progressive hanging wall caving at Chambishi copper mine in Zambia using the base friction model concept. Dissertation of Master Degree. London: London University, 1973

2 Ashiby J. Sliding and toppling modes of failure in model and jointed rock slopes. Dissertation of Master Degree. London: Royal School of Mines, 1971

3 Hittinger M. Numerical analysis of toppling failure in jointed rock. Dissertation of Doctor Degree. Berkeley: University of California, 1978

4 Bray J W, Goodman R E. 1981. The theory of base friction models. Int J Rock Mech Min, 1981, 18: 453-468

5 Adhikary D P, Dyskin A V, Jewell R J, et al. A study of mechanism of flexural topping failure of rock slopes. Rock Mech Rock Eng, 1997, 30: 75-93

6 Zhang J H, Chen Z Y, Wang X G. Centrifuge modeling of rock slopes susceptible to block toppling. Rock Mech Rock Eng, 2007, 40: 363-382

7 Pritchard M A, Savigny K W. Numerical modeling of toppling. Can Geotech J, 1990, 27: 824-834

8 Lanaro F, Jing L, Stephansson O, et al. D.E.M. modeling of laboratory tests of block toppling. Int J Rock Mech Min, 1997, 34: 173

9 Barla G, Devin P, Zaninetti A. Validation of a distinct element model for toppling rock slopes. In: 8th ISRM Congress. Int. Cong, on Rock Mechanics of Int. Society for Rock Mechanics, Tokyo, 1995, 3: $417-421$

10 Nichol S L, Hungr O, Evans S G. Large-scale brittle and ductile toppling of rock slopes. Can Geotech J, 2002, 39: 773-788

11 Goricki A, Goodman R E. Failure models of rock slopes demonstrated with base friction and simple numerical models. Felsbau, 2003, 21: $8-12$

12 Koo C Y, Chern J C. Modification of the DDA method for rigid block problems. I Int J Rock Mech Min, 1998, 35: 683-693

13 Wu J H, Ohnishi Y Z, Shi G H, et al. Theory of three-dimensional discontinuous deformation analysis and its application to a slope toppling at Amatoribashi, Japan. Int J Geomec ASCE, 2005, 5: 179-195

14 Shi G H. Applications of Discontinuous Deformation Analysis (DDA) to Rock Engineering. Beijing: Tsinghua University Press \& Springer, 2007. 136-147
15 Alzo'ubi A K, Martin C D, Cruden D M. Influence of tensile strength on toppling failure in centrifuge tests. Int J Rock Mech Min, 2010, 47: 974-982

16 Gu J, Zhao Z Y. Considerations of the discontinuous deformation analysis on wave propagation. Int J Numer Anal Meth Geomech, 2009, 33: 1449-1465

17 Scholtès L, Donzé F V. Modeling progressive failure in fratured rock masses using a 3D discrete element method. Int J Rock Mech Min, 2012, 52: 18-30

18 Ning Y J, Yang J, An X M, et al. Modeling rock fracturing and blast-induced rock mass failure via advanced discretisation within the discontinuous deformation analysis framework. Comput Geotech, 2011, 38: 40-49

19 Eberhardt E, Stead D, Coggan J S. Numerical analysis of initiation and progressive failure in natural rock slopes-the 1991 Randa rockslide. Int J Rock Mech Min, 2004, 41: 69-87

20 Zhang G X, Zhao Y, Peng X C. Simulation of toppling failure of rock slope by numerical manifold method. Int Comput Method, 2010, 7: 167-189

21 Feng C, Li S, Liu X, et al. A semi-spring and semi-edge combined contact model in CDEM and its application to analysis of Jiweishan landslide. J Rock Mech Geotec Eng, 2014, 6: 26-35

22 Schofield A N. Cambridge geotechnical centrifuge operations. Géotechnique, 1980, 30: 227-268

23 Kutter B L, Moquette L, Thompson P Y. Gravity-scaled tests on blast-induced soil-structure interaction. J Geotec Eng, 1988, 114: 431-447

24 Chen Z Y, Zhang J H, Wang W X, et al. Centrifuge modeling for rock slopes. In: $\mathrm{Ng} \mathrm{C}$, Zhang L M, Wang Y H, eds. Proceedings of the Sixth International Conference Physical Modelling Geotechnics, Hong kong, London: Taylor \& Francis Group, 2006. 19-28

25 Li S H, Zhao M H, Wang Y N, et al. A new numerical method for DEM-block and particle model. Int J Rock Mech Min, 2004, 41: 414-418

26 Ma Z S, Feng C, Liu T P, et al. A GPU accelerated continuousbaseddiscrete element method for elasto dynamics analysis. Adv Mater Res, 2011, 320: 329-334

27 Wang J, Li S H, Feng C. A shrunken edge algorithm for contact detection between convex polyhedral blocks. Comput Geotech, 2015, 63: $315-330$

28 Shi G H. Discontinuous deformation analysis-Anew model for the statics and dynamics of block systems. Dissertation of Doctor Degree. Berkeley: University of California, 1988

29 Shi G H. Simplex integration for manifold method and discontinuous deformation analysis. In: Working Forum on the Manifold Method of Material Analysis, Chungli, Taiwan, 1995. 2, 129-164

30 Jing L. Formulation of discontinuous deformation analysis (DDA) animplicit discrete element model for block systems. Eng Geol, 1998, 49: 371-381

31 Jing L. A review of techniques, advances and outstanding issues in numerical modeling fro rock mechanics and rock engineering. Int $\mathbf{J}$ Rock Mech Min, 2003, 40: 285-353

32 Dooling D M, Sitar N. Time integration in discontinuous deformation analysis. J Eng Mech-ASCE, 2004, 130: 249-259 